GOEING gCIENTIFIC RESEARCH LABCPATOAIES DDCUMENT D1-Be-0EgO Bum

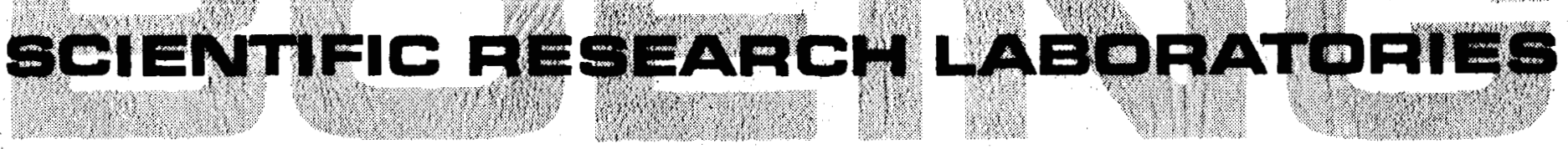

\title{
The Precession and Nutation of Deformable Bodies
}

\author{
Zdeněk Kopal
}
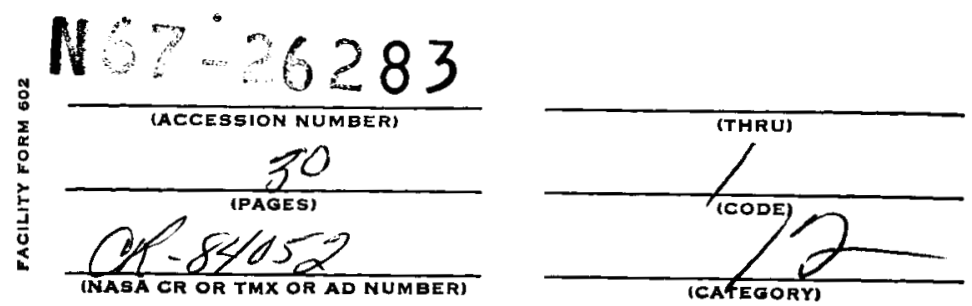
THE PRECESSION AND NUTATION OF DEFORMABLE BODIES

$$
\text { by }
$$

Zdeněk Kopal

This research was supported in part by the National Aeronautics and Space Administration under Contract No. NASW-1470.

\footnotetext{
Mathematical Note No. 496

Mathematics Research Laboratory

BOEING SCIENTIFIC RESEARCH LABORATORIES
}

December 1966 
ABSTRACT

The aim of the present communication has been to set up the Eulerian system of equations which governs the motion of a self-gravitating deformable body (regarded as a compressible fluid of arbitrarily high viscosity) about its own center of gravity in an arbitrary external field of force. If the latter were particularized to represent the tidal attraction of the Sun and the Moon, this motion would represent the luni-solar precession and nutation of a fluid Earth; if, on the other hand, the external field of force were governed by the Earth (or the Sun), the motion would define the physical librations of the Moon regarded as a deformable body. All these (and other) cases arising in the solar system will be treated in due course.

The specific aim of this first of a series of reports in which these problems will be discussed will be to establish the explicit form of the system of differential equations which are basic to our problem. One specific aspect of their solution--namely, dynamical tides on deformable bodies and the consequent dissipation of energy--will be deferred to a second report of this series; while reports III and IV will be concerned with particular cases of the precession and librations of the Earth and the Moon. The concluding report $\mathrm{V}$ will be devoted to a discussion of the differences which are encountered if the self-gravitating deformable body behaves, not as a viscous fluid, but rather as an elastic solid. As will be shown, these differences will prove to be largely formal, and limited mainly to a different interpretation of the parameters involved. 


\section{INTRODUCTION}

The differential equations which govern the motions of selfgravitating bodies about their center of gravity--whether free or forced--have been known since the early days of the history of rational mechanics; and the investigators of their solutions bearing on the precession and nutation of the Earth, or the physical librations of the Moon, included (to name only the greatest) Newton, Euler, Lagrange and Laplace. All these investigators assumed in common that the body moving about its center of gravity in an external field of force can be regarded as rigid; and its external form (or moments of inertia) be fixed and independent of the time. However, it was not till in the second half of the 19 th century that it has been gradually realized that a selfgravitating body of the mass of the Earth or the Moon cannot be regarded as rigid or incompressible; moreover, observations have revealed (at least in the case of the Earth) that its form responds to a fluctuating external field of force through bodily tides.

A mathematical treatment of the motion of deformable bodies about their center of mass in an external field of force was, however, slow to come (cf. Liouvi1le, 1858; Gyldén, 1871; Oppenheimer, 1885; Darwin, 1879; Poincaré, 1910) and is still far from being solved for the precession or nutation of the Earth; while its bearing on the physical librations of the lunar globe has not yet even been considered. The aim of the present series of reports will be to provide a more comprehensive treatment of this subject than has been done by all previous investigators, 
and to do so on the basis of the fundamental equations of hydrodynamics, in which the three velocity components $u, v, w$ will be systematically expressed in terms of the independent rotations, about the three respective axes $x, y, z$, with angular velocities $\omega_{x}, \omega_{y}$, and $\omega_{z}$. Departures from a hydrodynamical treatment, necessitated if the response of a deformable body to an external strain is that of an elastic solid rather than that of a viscous fluid, (i.e., if we deal with a "Maxwel1" rather than "Kelvin-Voigt" body), will be taken up in the concluding report of this series.

\section{EQUATIONS OF THE PROBLEM}

As is we11 known, the Eulerian fundamental equations of hydrodynamics governing the motion of compressible viscous fluids can be expressed in rectangular coordinates in the symmetrical form

$$
\begin{aligned}
& \rho \frac{D u}{D t}=\rho \frac{\partial \Omega}{\partial x}-\frac{\partial P}{\partial x}+\frac{\partial \sigma x x}{\partial x}+\frac{\partial \sigma_{x y}}{\partial y}+\frac{\partial \sigma x z}{\partial z}, \\
& \rho \frac{D v}{D t}=\rho \frac{\partial \Omega}{\partial y}-\frac{\partial P}{\partial y}+\frac{\partial \sigma y x}{\partial x}+\frac{\partial \sigma y y}{\partial y}+\frac{\partial \sigma y z}{\partial z} \text {, } \\
& \rho \frac{D W}{D t}=\rho \frac{\partial \Omega}{\partial z}-\frac{\partial P}{\partial z}+\frac{\partial \sigma_{z x}}{\partial x}+\frac{\partial \sigma_{z y}}{\partial y}+\frac{\partial \sigma_{z z}}{\partial z},
\end{aligned}
$$

where $u, v, w$ denote the velocity components of fluid motion, at the time $t$, in the direction of increasing coordinates $x, y, z$, respectively;

$$
\frac{D}{D t} \equiv \frac{\partial}{\partial t}+u \frac{\partial}{\partial x}+v \frac{\partial}{\partial y}+w \frac{\partial}{\partial z}
$$

representing the Lagrangian time-derivative (following the motion); $\rho$ stands for the local density of the fluid; P, for its pressure; $\Omega$, for 
the total potential (internal as well as external) of all forces acting upon it; and

$$
\begin{aligned}
& \sigma_{x x}=\frac{2}{3} \mu\left\{3 \frac{\partial u}{\partial x}-\Delta\right\}, \\
& \sigma_{y y}=\frac{2}{3} \mu\left\{3 \frac{\partial v}{\partial y}-\Delta\right\}, \\
& \sigma_{z z}=\frac{2}{3} \mu\left\{3 \frac{\partial w}{\partial z}-\Delta\right\}, \\
& \sigma_{x y}=\mu\left\{\frac{\partial v}{\partial x}+\frac{\partial u}{\partial y}\right\}=\sigma_{y x}, \\
& \sigma_{y z}=\mu\left\{\frac{\partial w}{\partial y}+\frac{\partial v}{\partial z}\right\}=\sigma_{z y}, \\
& \sigma_{z x}=\mu\left\{\frac{\partial u}{\partial z}+\frac{\partial w}{\partial x}\right\}=\sigma_{x z},
\end{aligned}
$$

are the respective components of the viscous stress tensor, where $\mu$ denotes the coefficient of viscosity and

$$
\Delta \equiv \frac{\partial u}{\partial x}+\frac{\partial v}{\partial y}+\frac{\partial w}{\partial z}
$$

the divergence of the velocity vector of the fluid.

As is well known, equations (2-1) - (2-3) safeguard the conservation of momentum of the underlying dynamical problem; and as such represent only one-half of the system necessary for a complete specification of the six dependent variables

$$
\begin{aligned}
& u, v, w ; \\
& \rho, P, \Omega ;
\end{aligned}
$$

of our problem. Of the remaining three equations, two can be adjoined with relative ease: namely, the equation of continuity 


$$
\frac{D \rho}{D t}+\rho \Delta=0
$$

safeguarding the conservation of mass, and the Poisson equation

$$
\nabla^{2} \Omega=-4 \pi G \rho
$$

which must be satisfied by the gravitational potential (G denoting the constant of gravitation).

The sole remaining equation required to render the solution of our system determinate (for an appropriate set of boundary conditions) must be derived from the principle of the conservation of energy, in the form of an "equation of state" relating $\mathrm{P}$ and $\rho$; but its explicit formulation will be postponed until a later stage of our analysis.

\section{THE COMPONENTS OF VELOCITIES AND ACCELERATIONS}

In order to apply the system of equations set up in the preceding section for the study of the motion of a self-gravitating body about its center of gravity, consider the transformation of rectangular coordinates between an inertial (fixed) system of space axes $x, y, z$, and a rotating system of body (primed) axes $x^{\prime}, y^{\prime}, z^{\prime}$, possessing the same origin, but with the primed axes rotated with respect to the space axes by the Eulerian angles $\phi, \theta, \psi$, in accordance with a scheme illustrated on the accompanying Figure $3-1$.

As is well known, the transformation of coordinates from the space to the body axes is governed by the following matrix equation 


\section{$-5-$}

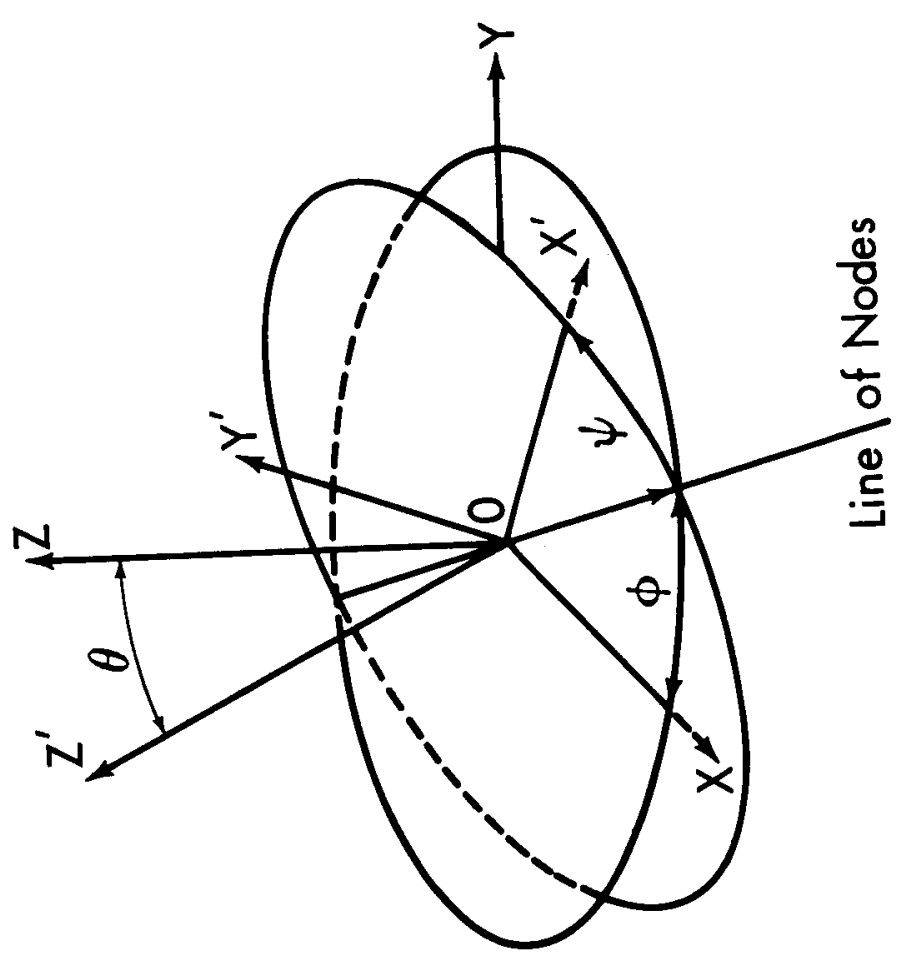

Fig. 3-1 


$$
\left\{\begin{array}{l}
x \\
y \\
z
\end{array}\right)=\left(\begin{array}{ccc}
a_{11} & a_{12} & a_{13} \\
a_{21} & a_{22} & a_{23} \\
a_{31} & a_{32} & a_{33}
\end{array}\right\}\left\{\begin{array}{l}
x^{\prime} \\
y^{\prime} \\
z^{\prime}
\end{array}\right\},
$$

where the coefficients $a_{i k}$, expressed in terms of the Eulerian angles assume the explicit forms

$$
\left.\begin{array}{l}
a_{11}=\cos \psi \cos \phi-\cos \theta \sin \phi \sin \psi, \\
a_{12}=-\sin \psi \cos \phi-\cos \theta \sin \phi \cos \psi, \\
a_{13}=\sin \theta \sin \phi \quad ;
\end{array}\right\}
$$

In order to obtain the corresponding space velocity-components $u, v, w$, let us differentiate equations (3-1) with respect to the time. With dots denoting hereafter ordinary (total) derivatives with respect to $t$, we find that

$$
\begin{aligned}
& \dot{x}=u=\dot{a}_{11} x^{\prime}+\dot{a}_{12} y^{\prime}+\dot{a}_{13} z^{\prime}+a_{11} \dot{x}^{\prime}+a_{12} \dot{y}^{\prime}+a_{13} \dot{z}^{\prime}, \\
& \dot{y}=v=\dot{a}_{21} x^{\prime}+\dot{a}_{22} y^{\prime}+\dot{a}_{23} z^{\prime}+a_{21} \dot{x}^{\prime}+a_{22} \dot{y}^{\prime}+a_{23} \dot{z}^{\prime}, \\
& \dot{z}=w=\dot{a}_{31} x^{\prime}+\dot{a}_{32} y^{\prime}+\dot{a}_{33} z^{\prime}+a_{31} \dot{x}^{\prime}+a_{32} \dot{y}^{\prime}+a_{33} \dot{z}^{\prime}
\end{aligned}
$$


whereas the body velocity-components $u^{\prime}, v^{\prime}, w^{\prime}$ follow from

$$
\begin{aligned}
\dot{x}^{\prime}=u^{\prime} & =\dot{a}_{11} x+\dot{a}_{21} y+\dot{a}_{31} z \\
& +a_{11} \dot{x}+a_{21} \dot{y}+a_{31} \dot{z}, \\
\dot{y}^{\prime}=v^{\prime} & =\dot{a}_{12} x+\dot{a}_{22} y+\dot{a}_{32} z \\
& +a_{12} \dot{x}+a_{22} \dot{y}+a_{32} \dot{z}, \\
\dot{z}^{\prime}=w^{\prime} & =\dot{a}_{13} x+\dot{a}_{23} y+\dot{a}_{33} z \\
& +a_{13} \dot{x}+a_{23} \dot{y}+a_{33} \dot{z} ;
\end{aligned}
$$

where

$$
\left.\begin{array}{rl}
\dot{a}_{11}=a_{12} \dot{\psi}-a_{21} \dot{\phi}+a_{31} \dot{\theta} \sin \phi & =a_{31} \omega_{y}-a_{21} \omega_{z} \\
& =a_{12} \omega_{z^{\prime}}-a_{13} \omega_{y^{\prime}},
\end{array}\right\}
$$




$$
\left.\begin{array}{rl}
\dot{a}_{31}=a_{32} \dot{\psi} \quad+\dot{\theta} \sin \psi \cos \theta & =a_{21} \omega_{x}-a_{11} \omega_{y} \\
& =a_{32} \omega_{z^{\prime}}-a_{33} \omega_{y^{\prime}},
\end{array}\right\}
$$

where, taking advantage of the fact that

$$
\left.\begin{array}{l}
a_{11} \dot{a}_{11}+a_{12} \dot{a}_{12}+a_{13} \dot{a}_{13}=0 \\
a_{21} \dot{a}_{21}+a_{22} \dot{a}_{22}+a_{23} \dot{a}_{23}=0 \\
a_{31} \dot{a}_{31}+a_{32} \dot{a}_{32}+a_{33} \dot{a}_{33}=0
\end{array}\right\}
$$

and

$$
\left.\begin{array}{l}
a_{11} \dot{a}_{11}+a_{21} \dot{a}_{21}+a_{31} \dot{a}_{31}=0 \\
a_{12} \dot{a}_{12}+a_{22} \dot{a}_{22}+a_{32} \dot{a}_{32}=0 \\
a_{13} \dot{a}_{13}+a_{23} \dot{a}_{23}+a_{33} \dot{a}_{33}=0
\end{array}\right\}
$$

the respective angular velocities of rotation are given by

$$
\begin{aligned}
w_{x} & =+\left(a_{21} \dot{a}_{31}+a_{22} \dot{a}_{32}+a_{23} \dot{a}_{33}\right) \\
& =-\left(a_{31} \dot{a}_{21}+a_{32} \dot{a}_{22}+a_{33} \dot{a}_{23}\right), \\
w_{y} & =+\left(a_{31} \dot{a}_{11}+a_{32} \dot{a}_{12}+a_{33} \dot{a}_{13}\right) \\
& =-\left(a_{11} \dot{a}_{31}+a_{12} \dot{a}_{32}+a_{13} \dot{a}_{33}\right), \\
w_{z} & =+\left(a_{11} \dot{a}_{21}+a_{12} \dot{a}_{22}+a_{13} \dot{a}_{23}\right) \\
& =-\left(a_{21} \dot{a}_{11}+a_{22} \dot{a}_{12}+a_{23} \dot{a}_{13}\right),
\end{aligned}
$$


with respect to the space axes; or

$$
\left.\begin{array}{rl}
w_{x^{\prime}} & =+\left(a_{13} \dot{a}_{12}+a_{23} \dot{a}_{22}+a_{33} \dot{a}_{32}\right) \\
& =-\left(a_{12} \dot{a}_{13}+a_{22} \dot{a}_{23}+a_{32} \dot{a}_{33}\right), \\
\omega_{y^{\prime}} & =+\left(a_{11} \dot{a}_{13}+a_{21} \dot{a}_{23}+a_{31} \dot{a}_{33}\right) \\
& =-\left(a_{13} \dot{a}_{11}+a_{23} \dot{a}_{21}+a_{33} \dot{a}_{31}\right),
\end{array}\right\}
$$

with respect to the body axes; the patrs of alternative equations arising from the fact that, by a time-differentiation of the relations $a_{i j} a_{i k}=\delta_{j k}$ it follows that $a_{i j} \dot{a}_{i k}+a_{i k} \dot{a}_{i j}=0$.

Inserting in the equations (3-22) - (3-27) from (3-11) - (3-19) it follows that, in terms of the Eulerian angles,

$$
\begin{aligned}
& \omega_{x}=\dot{\theta} \cos \phi+\dot{\psi} \sin \theta \sin \phi, \\
& \omega_{y}=\dot{\theta} \sin \phi-\dot{\psi} \sin \theta \cos \phi, \\
& \omega_{z}=\quad+\dot{\psi} \cos \theta+\dot{\phi} ;
\end{aligned}
$$

while

$$
\begin{aligned}
& \omega_{x^{\prime}}=\dot{\phi} \sin \theta \sin \psi+\dot{\theta} \cos \psi, \\
& \omega_{y^{\prime}}=\dot{\phi} \sin \theta \cos \psi-\dot{\theta} \sin \psi, \\
& \omega_{z^{\prime}}=\dot{\phi} \cos \theta \quad+\dot{\psi},
\end{aligned}
$$

as could be also directly verified by an application of the inverse of the transformation (3-1), in accordance with which 


$$
\begin{aligned}
& \omega_{x^{\prime}}=a_{11} \omega_{x}+a_{21} \omega_{y}+a_{31} \omega_{z}, \\
& \omega_{y^{\prime}}=a_{12} \omega_{x}+a_{22} \omega_{y}+a_{32} \omega_{z}, \\
& \omega_{z^{\prime}}=a_{13} \omega_{x}+a_{23} \omega_{y}+a_{33} \omega_{z} .
\end{aligned}
$$

With the aid of the preceding results the equations (3-5) - (3-7) or (3-8) - (3-10) for the velocity-components with respect to the space or body axes can be reduced to the forms

$$
\begin{aligned}
& u=z \omega_{y}-y \omega_{z}+u_{0}^{\prime} \\
& v=x \omega_{z}-z \omega_{x}+v_{0}^{\prime} \\
& w=y \omega_{x}-x \omega_{y}+w_{0}^{\prime}
\end{aligned}
$$

or

$$
\begin{aligned}
& u^{\prime}=-z^{\prime} \omega_{y^{\prime}}+y^{\prime} \omega_{z^{\prime}}+u_{0}, \\
& v^{\prime}=-x^{\prime} \omega_{z^{\prime}}+z^{\prime} \omega_{x^{\prime}}+v_{0}, \\
& w^{\prime}=-y^{\prime} \omega_{x^{\prime}}+x^{\prime} \omega_{y^{\prime}}+w_{0},
\end{aligned}
$$

where

$$
\begin{aligned}
& u_{0}=a_{11} u+a_{21} v+a_{31} w \\
& v_{0}=a_{12} u+a_{22} v+a_{32} w \\
& w_{0}=a_{13} u+a_{23} v+a_{33} w
\end{aligned}
$$

are the space velocity components in the direction of the rotating axes $x^{\prime}, y^{\prime}, z^{\prime} ;$ and

$$
\begin{aligned}
& u_{0}^{\prime}=a_{11} u^{\prime}+a_{12} v^{\prime}+a_{13} w^{\prime} \\
& v_{0}^{\prime}=a_{21} u^{\prime}+a_{22} v^{\prime}+a_{23} w^{\prime} \\
& w_{0}^{\prime}=a_{31} u^{\prime}+a_{32} v^{\prime}+a_{33} w^{\prime}
\end{aligned}
$$

are the body velocity components in the direction of the fixed axes 
$x, y, z$.

In order to specify the appropriate forms of the components of acceleration, let us differentiate the foregoing expressions (3-35) (3-40) for the velocity components with respect to the time. Doing so we find "that those with respect to the space axes assume the forms

$$
\begin{aligned}
& \dot{\mathrm{u}}=w \omega_{\mathrm{y}}+z \dot{\omega}_{\mathrm{y}}-\mathrm{v} \omega_{z}-\mathrm{y} \dot{\omega}_{z}+\dot{\mathrm{u}}_{0}, \\
& \dot{\mathrm{v}}=\mathrm{u} \omega_{\mathrm{z}}+\mathrm{x} \dot{\omega}_{z}-\mathrm{w} \omega_{\mathrm{x}}-\mathrm{z} \dot{\omega}_{\mathrm{x}}+\dot{\mathrm{v}}_{0}, \\
& \dot{\mathrm{w}}=\mathrm{v} \omega_{\mathrm{x}}+\mathrm{y} \dot{\omega}_{\mathrm{x}}-\mathrm{u} \omega_{\mathrm{y}}-\mathrm{x} \dot{\omega}_{\mathrm{y}}+\dot{\mathrm{w}}_{0},
\end{aligned}
$$

where the velocity components $\mathrm{u}, \mathrm{v}$, $\mathrm{w}$ have already been given by equations (3-35) - (3-37); and where, by differentiation of (3-42),

$$
\begin{aligned}
\dot{u}_{0}^{\prime}=a_{11} \dot{u}^{\prime} & +a_{12} \dot{v}^{\prime}+a_{13 \dot{w}^{\prime}} \\
& +\dot{a}_{11} u^{\prime}+\dot{a}_{12} v^{\prime}+\dot{a}_{13} w^{\prime}, \\
\dot{v}_{0}^{\prime}=a_{21} \dot{u}^{\prime} & +a_{22} \dot{v}^{\prime}+a_{23} \dot{w}^{\prime} \\
& +\dot{a}_{21} u^{\prime}+\dot{a}_{22} v^{\prime}+\dot{a}_{23 w^{\prime}}, \\
\dot{w}_{0}^{\prime}=a_{31} \dot{u}^{\prime} & +a_{32} \dot{v}^{\prime}+a_{33} \dot{w}^{\prime} \\
& +\dot{a}_{31} u^{\prime}+\dot{a}_{32} v^{\prime}+\dot{a}_{33} w^{\prime} .
\end{aligned}
$$

The first three terms in each of these expressions represent obviously the body accelerations with respect to the space axes; and we shall abbreviate them as

$$
\begin{aligned}
& a_{11} \dot{u}^{\prime}+a_{12} \dot{v}^{\prime}+a_{13} \dot{w}^{\prime}=(\dot{u})_{0}^{\prime}, \\
& a_{21} \dot{u}^{\prime}+a_{22} \dot{v}^{\prime}+a_{23} \dot{w}^{\prime}=(\dot{v})_{0}^{\prime}, \\
& a_{31} \dot{u}^{\prime}+a_{32} \dot{v}^{\prime}+a_{33} \dot{w}^{\prime}=(\dot{w})_{0}^{\prime} .
\end{aligned}
$$


Since, moreover, by insertion from (3-11) - (3-13) and (3-42),

$$
\begin{aligned}
& \dot{a}_{11} u^{\prime}+\dot{a}_{12} v^{\prime}+\dot{a}_{13} w^{\prime}=\left(a_{31} \omega_{y}-a_{21} \omega_{z}\right) u^{\prime} \\
&+\left(a_{32} \omega_{y}-a_{22} \omega_{z}\right) v^{\prime} \\
&+\left(a_{33} \omega_{y}-a_{23} \omega_{z}\right) w^{\prime} \\
&=\omega_{y}\left(a_{31} u^{\prime}+a_{32} v^{\prime}+a_{33} w^{\prime}\right) \\
&-\omega_{z}\left(a_{21} u^{\prime}+a_{22} v^{\prime}+a_{23} w^{\prime}\right) \\
&=\omega_{y} w_{0}^{\prime}-\omega_{z} v_{0}^{\prime} ;
\end{aligned}
$$

and, similarly,

$$
\dot{a}_{21} u^{\prime}+\dot{a}_{22} v^{\prime}+\dot{a}_{23} w^{\prime}=\omega_{z} u_{0}^{\prime}-\omega_{x} w_{0}^{\prime}
$$

while

$$
\dot{a}_{31} u^{\prime}+\dot{a}_{32} v^{\prime}+\dot{a}_{33} w^{\prime}=w_{x} v_{0}-\omega_{y} u_{0}^{\prime},
$$

equations (3-43) - (3-45) can be rewritten in a more explicit form

$$
\begin{aligned}
\dot{u}=-x\left(\omega_{y}^{2}+\omega_{z}^{2}\right)+y\left(\omega_{x} \omega_{y}-\dot{\omega}_{z}\right) & +z\left(\omega_{x} \omega_{z}+\dot{\omega}_{y}\right) \\
& +(\dot{u}) \delta+2\left(\omega_{0}^{\prime} \omega_{y}-v j \omega_{z}\right), \\
\dot{v}=-y\left(\omega_{z}^{2}+\omega_{x}^{2}\right)+z\left(\omega_{y} \omega_{z}-\dot{\omega}_{x}\right) & +x\left(\omega_{x} \omega_{y}+\dot{\omega}_{z}\right) \\
& +\left(\dot{v}_{0}^{\prime}+2\left(u_{0}^{\prime} \omega_{z}-\omega_{0}^{\prime} \omega_{x}\right),\right.
\end{aligned}
$$

and

$$
\begin{aligned}
\dot{w}=-z\left(\omega_{x}^{2}+\omega_{y}^{2}\right)+x\left(\omega_{x} \omega_{z}-\dot{\omega}_{y}\right) & +y\left(\omega_{y} \omega_{z}+\dot{\omega}_{x}\right) \\
& +(\dot{w})_{0}^{\prime}+2\left(v_{0}^{\prime} \omega_{x}-u_{0}^{\prime} \omega_{y}\right) .
\end{aligned}
$$

The foregoing equations refer to accelerations with respect to the inertial system of space axes. Those with respect to the (rotating) body axes can be obtained by an analogous process from the equations 


$$
\begin{aligned}
& \dot{u}^{\prime}=-w^{\prime} \omega_{y^{\prime}}-z^{\prime} \dot{w}_{y^{\prime}}+v^{\prime} \omega_{z^{\prime}}+y^{\prime} \dot{w}_{z^{\prime}}+\dot{u}_{0}, \\
& \dot{v}^{\prime}=-u^{\prime} \omega_{z^{\prime}}-x^{\prime} \dot{w}_{z^{\prime}}+w^{\prime} \omega_{x^{\prime}}+z^{\prime} \dot{w}_{x^{\prime}}+\dot{v}_{0}, \\
& \dot{w}^{\prime}=-v^{\prime} \omega_{x^{\prime}}-y^{\prime} \dot{\omega}_{x^{\prime}}+u^{\prime} \omega_{y^{\prime}}+x^{\prime} \dot{w}_{y^{\prime}}+\dot{w}_{0},
\end{aligned}
$$

equivalent to (3-43) - (3-45); which on being treated in the same way as the latter can eventually be reduced to the form

$$
\begin{aligned}
\dot{u}^{\prime}=-x^{\prime}\left(\omega_{y^{\prime}}^{2}+\omega_{z^{\prime}}^{2}\right) & +y^{\prime}\left(\omega_{x^{\prime}} \omega_{y^{\prime}}+\dot{\omega}_{z^{\prime}}\right)+z^{\prime}\left(\omega_{x^{\prime}} \omega_{z^{\prime}}-\dot{\omega}_{y^{\prime}}\right) \\
& +(\dot{u})_{0}-2\left(w_{0} \omega_{y^{\prime}}-v_{0} \omega_{z^{\prime}}\right), \\
\dot{v}^{\prime}=-y^{\prime}\left(\omega_{z^{\prime}}^{2}+\omega_{x^{\prime}}\right) & +z^{\prime}\left(\omega_{y^{\prime}} \omega_{z^{\prime}}+\dot{\omega}_{x^{\prime}}\right)+x^{\prime}\left(\omega_{x^{\prime}} \omega_{y^{\prime}}-\dot{\omega}_{z^{\prime}}\right) \\
& +(\dot{v})_{0}-2\left(u_{0} \omega_{z^{\prime}}-w_{0} \omega_{x^{\prime}}\right), \\
\dot{w}^{\prime}=-z^{\prime}\left(\omega_{x^{\prime}}^{2}+\omega_{y^{\prime}}\right) & +x^{\prime}\left(\omega_{x^{\prime}} \omega_{z^{\prime}}+\dot{\omega}_{y^{\prime}}\right)+y^{\prime}\left(\omega_{y^{\prime}} \omega_{z^{\prime}}-\dot{\omega}_{x^{\prime}}\right) \\
& +(\dot{w})_{0}-2\left(v_{0} \omega_{x^{\prime}}-u_{0} \omega_{y^{\prime}}\right),
\end{aligned}
$$

where the space velocity components $u_{0}, v_{0}, w_{0}$ in the direction of increasing $x^{\prime}, y^{\prime}, z^{\prime}$ continue to be given by equations (3-41); while the corresponding components of the accelerations are given by

$$
\begin{aligned}
& (\dot{\mathrm{u}})_{0}=\mathrm{a}_{11} \dot{\mathrm{u}}+\mathrm{a}_{21} \dot{\mathrm{v}}+\mathrm{a}_{31} \dot{\mathrm{w}}, \\
& (\dot{\mathrm{v}})_{0}=\mathrm{a}_{12} \dot{\mathrm{u}}+\mathrm{a}_{22} \dot{\mathrm{v}}+\mathrm{a}_{32} \dot{\mathrm{w}}, \\
& (\dot{\mathrm{w}})_{0}=\mathrm{a}_{13} \dot{\mathrm{u}}+\mathrm{a}_{23} \dot{\mathrm{v}}+\mathrm{a}_{33} \dot{\mathrm{w}} .
\end{aligned}
$$

If, in particular, we consider the restricted case of a rotation about the z-axis only (so that $\omega_{x}=\omega_{y}=0$ ), equations $(3-53)-(3-55)$ will reduce to the system

$$
\begin{aligned}
& \dot{\mathrm{u}}=(\dot{\mathrm{u}})_{0}^{\prime}-2 \mathrm{v} \omega_{z}+\mathrm{x} \omega_{z}^{2}-y \dot{\omega}_{z}, \\
& \dot{\mathrm{v}}=(\dot{\mathrm{v}}) \dot{0}+2 \mathrm{u} \omega_{z}+\mathrm{y} \omega_{z}^{2}+\mathrm{x} \dot{\omega}_{z}, \\
& \dot{\mathrm{w}}=(\dot{\mathrm{w}}) \dot{0} ;
\end{aligned}
$$


while equations (3-59) - (3-61) will likewise reduce to

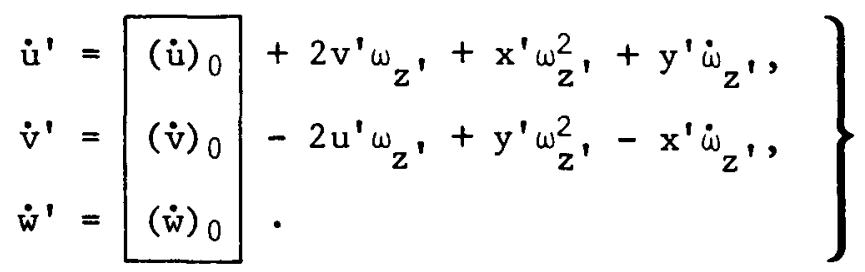

It is the accelerations in the cartouches of the two systems--referred as they are to the inertial space axes--which should be identified with the Lagrangian time-derivatives

$$
\frac{D \vec{V}}{D t}
$$

on the left-hand sides of the equations $(2-2)-(2-3)$ of motion if these are referred to the inertial or rotating axes of coordinates.

A closing note concerning the time differentiation of the coordinates or velocities should be added in this place. As

$$
x \equiv x(t), \quad y \equiv y(t), \quad z \equiv z(t),
$$

it follows that

$$
\begin{aligned}
& \dot{x} \equiv u=\frac{d x}{d t}=\frac{\partial x}{\partial t}, \\
& \dot{y} \equiv v=\frac{d y}{d t}=\frac{\partial y}{\partial t}, \\
& \dot{z} \equiv w=\frac{d y}{d t}=\frac{\partial z}{\partial t},
\end{aligned}
$$

i.e., the ordinary (total) and partial derivatives of the coordinates with respect to the time are obviously identical. This is, however, no longer true of the time-differentiation of the velocities--whether 
linear or angular. As

$$
\begin{aligned}
& u \equiv u(x, y, z ; t) \\
& v \equiv v(x, y, z ; t) \\
& w \equiv w(x, y, z ; t)
\end{aligned}
$$

or

$$
\omega_{x, y, z} \equiv \omega_{x, y, z}(x, y, z ; t),
$$

where the coordinates (3-65) are themselves functions of the time. In consequence,

$$
\begin{aligned}
\dot{u} \equiv \frac{d u}{d t} & =\frac{\partial u}{\partial t}+\frac{\partial u}{\partial x} \frac{\partial x}{\partial t}+\frac{\partial u}{\partial y} \frac{\partial y}{\partial t}+\frac{\partial u}{\partial z} \frac{\partial z}{\partial t} \\
& =\frac{\partial u}{\partial t}+u \frac{\partial u}{\partial x}+v \frac{\partial u}{\partial y}+w \frac{\partial u}{\partial z}
\end{aligned}
$$

by virtue of (3-66); and similarly for $\dot{v}$ and $\dot{w}$. Likewise,

$$
\dot{\omega} \equiv \frac{d \omega}{d t}=\frac{\partial \omega}{\partial t}+u \frac{\partial \omega}{\partial x}+v \frac{\partial \omega}{\partial y}+w \frac{\partial \omega}{\partial z}
$$

for $\omega \equiv \omega_{\mathrm{x}, \mathrm{y}, \mathrm{z}}$.

For coordinate systems referred to the rotating body axes similar reltions hold good; care being merely taken to replace the unprimed coordinates or velocity components by the primed ones.

IV. FORMATION OF THE EULERIAN EQUATIONS FOR PRECESSION AND NUTATION

In Section II of this report we set up the general form of the equations governing the motion of compressible viscous fluids in rectangular coordinates; and in Section III we expressed its velocity components in 
terms of arbitrary rotations about three rectangular axes. The aim of the present section will be to combine the fundamental equations (2-1) (2-3) rewritten in terms of the angular variables $\omega_{x, y, z}$ introduced in Section III in a form suitable for their subsequent solution.

In order to embark on this task, let us multiply equations (3-53) (3-55) by $x, y, z$ and form their following differences:

$$
\begin{aligned}
& y \dot{w}-z \dot{v}=\left(y^{2}+z^{2}\right) \dot{\omega}_{x}+\left(y^{2}-z^{2}\right) \omega_{y} \omega_{z} \\
& -x y\left(\dot{\omega}_{y}-\omega_{x} \omega_{z}\right)-x z\left(\dot{\omega}_{z}+\omega_{x} \omega_{y}\right) \\
& -y z\left(\omega_{y}^{2}-\omega_{z}^{2}\right) \\
& +\left\{y(\dot{w}) j_{0}^{\prime}-z(\dot{v}) j_{0}^{\prime}\right\}+2 y\left\{v_{0}^{\prime} \omega_{x}-u_{0}^{\prime} \omega_{y}\right\} \\
& -2 z\left\{u_{0}^{\prime} \omega_{z}-w_{0}^{\prime} \omega_{x}\right\} \text {, } \\
& z \dot{u}-x \dot{w}=\left(z^{2}+x^{2}\right) \dot{\omega}_{y}+\left(z^{2}-x^{2}\right) \omega_{x} \omega_{z} \\
& -y z\left(\dot{\omega}_{z}-\omega_{y} \omega_{x}\right)-y x\left(\dot{\omega}_{x}+\omega_{y} \omega_{z}\right) \\
& -\operatorname{zx}\left(\omega_{z}^{2}-\omega_{x}^{2}\right) \\
& +\left\{z(\dot{u}) j_{0}^{\prime}-x(\dot{w}) \dot{0}_{0}\right\}+2 z\left\{w_{0}^{\prime} \omega_{y}-v_{0}^{\prime} \omega_{z}\right\} \\
& -2 x\left\{v_{0}^{\prime} \omega_{x}-u_{0}^{\prime} \omega_{y}\right\} \text {, } \\
& x \dot{v}-y \dot{u}=\left(x^{2}+y^{2}\right) \dot{\omega}_{z}+\left(x^{2}-y^{2}\right) \omega_{x} \omega_{y} \\
& -z x\left(\dot{\omega}_{x}-\omega_{y} \omega_{z}\right)-z y\left(\dot{\omega}_{y}+\omega_{x} \omega_{z}\right) \\
& -x y\left(\omega_{x}^{2}-\omega_{y}^{2}\right) \text {, } \\
& +\left\{x(\dot{v}){ }_{0}^{\prime}-y(\dot{u})_{0}^{\prime}\right\}+2 x\left\{u_{0}^{\prime} \omega_{z}-w_{0}^{\prime} \omega_{x}\right\} \\
& -2 y\left\{w_{0}^{\prime} \omega_{y}-v_{0}^{\prime} \omega_{z}\right\} \text {. }
\end{aligned}
$$

If so, however, equations $(2-1)-(2-3)$ can be combined accordingly to yield 


$$
\begin{aligned}
& y \dot{w}-z \dot{v}+\frac{1}{\rho}\left\{y \frac{\partial}{\partial z}-z \frac{\partial}{\partial y}\right\} P-\left\{y \frac{\partial}{\partial z}-z \frac{\partial}{\partial y}\right\} \Omega=y \mathcal{H}-{ }_{z} \mathcal{G}, \\
& z \dot{u}-x \dot{w}+\frac{1}{\rho}\left\{z \frac{\partial}{\partial x}-x \frac{\partial}{\partial z}\right\} P-\left\{z \frac{\partial}{\partial x}-x \frac{\partial}{\partial z}\right\} \Omega={ }_{z} \mathcal{G}-x \mathcal{H}, \\
& x \dot{v}-y \dot{u}+\frac{1}{\rho}\left\{x \frac{\partial}{\partial y}-y \frac{\partial}{\partial x}\right\} P-\left\{x \frac{\partial}{\partial y}-y \frac{\partial}{\partial x}\right\} \Omega=x \mathcal{G}-y \mathcal{G},
\end{aligned}
$$

where

$$
\begin{aligned}
& \rho \mathcal{F}=\frac{\partial \sigma_{x x}}{\partial x}+\frac{\partial \sigma_{x y}}{\partial y}+\frac{\partial \sigma_{x z}}{\partial z}, \\
& \rho \mathcal{G}=\frac{\partial \sigma_{y x}}{\partial x}+\frac{\partial \sigma_{y y}}{\partial y}+\frac{\partial \sigma_{y z}}{\partial z}, \\
& \rho \mathcal{H}=\frac{\partial \sigma x}{\partial x}+\frac{\partial \sigma_{z y}}{\partial y}+\frac{\partial \sigma_{z z}}{\partial z},
\end{aligned}
$$

represent the effects of viscosity.

In order to proceed further, let us rewrite the foregoing expressions in terms of the respective velocity components. Inserting for the components $\sigma_{i j}$ of the viscous stress tensor from $(2-5)-(2-10)$ we find the expressions on the right-hand sides of equations (4-7) - (4-9) to assume the more explicit forms

$$
\begin{aligned}
\frac{\partial \sigma_{x x}}{\partial x}+\frac{\partial \sigma_{x y}}{\partial y}+\frac{\partial \sigma x z}{\partial z}= & \mu \nabla^{2} u+\frac{\mu}{3} \frac{\partial \Delta}{\partial x} \\
& +2\left\{\frac{\partial u}{\partial x}-\frac{\Delta}{3}\right\} \frac{\partial \mu}{\partial x} \\
& +\left\{\frac{\partial v}{\partial x}+\frac{\partial u}{\partial y}\right\} \frac{\partial \mu}{\partial y} \\
& +\left\{\frac{\partial u}{\partial z}+\frac{\partial w}{\partial x}\right\} \frac{\partial \mu}{\partial z},
\end{aligned}
$$




$$
\begin{aligned}
\frac{\partial \sigma}{\partial x}+\frac{\partial \sigma_{y y}}{\partial y}+\frac{\partial \sigma y z}{\partial z}= & \mu \nabla^{2} v+\frac{\mu}{3} \frac{\partial \Delta}{\partial y} \\
& +2\left\{\frac{\partial v}{\partial y}-\frac{\Delta}{3}\right\} \frac{\partial \mu}{\partial y} \\
& +\left\{\frac{\partial w}{\partial y}+\frac{\partial v}{\partial z}\right\} \frac{\partial \mu}{\partial z} \\
& +\left\{\frac{\partial v}{\partial x}+\frac{\partial u}{\partial y}\right\} \frac{\partial \mu}{\partial x},
\end{aligned}
$$

and

$$
\begin{aligned}
\frac{\partial \sigma_{z x}}{\partial x}+\frac{\partial \sigma_{z y}}{\partial y}+\frac{\partial \sigma_{z z}}{\partial z}= & \mu \nabla^{2} w+\frac{\mu}{3} \frac{\partial \Delta}{\partial z} \\
& +2\left\{\frac{\partial w}{\partial z}-\frac{\Delta}{3}\right\} \frac{\partial \mu}{\partial z} \\
& +\left\{\frac{\partial u}{\partial z}+\frac{\partial w}{\partial x}\right\} \frac{\partial \mu}{\partial x} \\
& +\left\{\frac{\partial w}{\partial y}+\frac{\partial v}{\partial z}\right\} \frac{\partial \mu}{\partial y},
\end{aligned}
$$

where $\Delta$ denotes, as before, the divergence (2-11) of the velocity vector; and $\nabla^{2}$ stands for the Laplacean operator.

Next, let us insert for the velocity components $u, v$, w from (3-35) - (3-37); by doing so we find that

$$
\begin{aligned}
& \nabla^{2} u=2 \nabla^{2} \omega_{y}-y \nabla^{2} \omega_{z}+\nabla^{2} u_{0}^{\prime}+2\left\{\frac{\partial \omega_{y}}{\partial z}-\frac{\partial \omega_{z}}{\partial y}\right\}, \\
& \nabla^{2} v=x \nabla^{2} \omega_{z}-z \nabla^{2} \omega_{x}+\nabla^{2} v_{0}^{\prime}+2\left\{\frac{\partial \omega_{z}}{\partial x}-\frac{\partial \omega_{x}}{\partial z}\right\}, \\
& \nabla^{2} w=y \nabla^{2} \omega_{x}-x \nabla^{2} \omega_{y}+\nabla^{2} w_{0}^{\prime}+2\left\{\frac{\partial \omega_{x}}{\partial y}-\frac{\partial \omega_{y}}{\partial x}\right\},
\end{aligned}
$$

and 


$$
\begin{aligned}
\Delta & =\left\{y \frac{\partial}{\partial z}-z \frac{\partial}{\partial y}\right\} \omega_{x}+\left\{z \frac{\partial}{\partial x}-x \frac{\partial}{\partial z}\right\} \omega_{y} \\
& +\left\{x \frac{\partial}{\partial y}-y \frac{\partial}{\partial x}\right\} \omega_{z}+\frac{\partial u_{0}^{\prime}}{\partial x}+\frac{\partial v_{0}^{\prime}}{\partial y}+\frac{\partial w_{0}^{\prime}}{\partial z} .
\end{aligned}
$$

Before proceeding further, one feature of basic importance should be brought out which we by-passed without closer discussion at an earlier stage: namely, when by virtue of equations (3-35) - (3-37) or (3-38)(3-40) we replaced the three dependent variables $u, v, w$ or $u^{\prime}, v^{\prime}, w^{\prime}$ on their left-hand sides by $s i x$ new variables $\omega_{x}, \omega_{y}, \omega_{z}$ and $u_{0}^{\prime}, v_{0}^{\prime}, w_{0}^{\prime}$ or $\omega_{x^{\prime}}, \omega_{y^{\prime}}, \omega_{z^{\prime}}$ and $u_{0}, v_{0}$, $w_{0}$ on their right-hand sides. This deliberately created redundancy permits us to impose without the loss of generality additional constraints on these variables, not embodied in the fundamental equations of Section II; and this we propose to do at the present time. We propose, in particular, to assume that the primed axes $x^{\prime} y^{\prime} z^{\prime}$ obtained by a rotation of the inertial system $x y z$, about a fixed origin, in accordance with the transformation (3-1) remain rectangular--an assumption to which implies, in effect, that the Eulerian angles $\theta, \phi, \psi$ involved in the direction cosines $a_{i k}$ and, therefore, in the angular velocity components $\omega_{x, y, z}$ or $\omega^{\omega^{\prime}, y^{\prime}, z^{\prime}}$ as defined by equations $(3-28)-(3-30)$ or $(3-31)-(3-33)$ are functions of the time $t$ alone (for should they depend, in addition, on the spatial coordinates $x, y, z$, a rotation as represented by equations (3-1) would result in a curvilinear coordinate system).

This assumption will neatly separate the physical meaning of the two groups of variables: for while the angular velocity components $\omega_{x, y, z}$ will describe a migid-body rotation of our dynamical system (during which 
the position of each particle remains unchanged in the primed coordinates), the remaining velocity components $u_{0}^{\prime}, v_{0}^{\prime}, w_{0}^{\prime}$ will represent deformation of our body, in the primed system, in the course of time. It is, therefore, the latter which will be of particular interest for the main problem which we have in mind; and in what follows, we propose to investigate the extent to which their occurrence may modify the structure of our equations.

In order to do so we notice first that, inasmuch as the angular velocity components are hereafter to be regarded as functions of $t$ alone it follows from $(4-13)-(4-15)$ that

$$
\left.\begin{array}{l}
\nabla^{2} \mathrm{u}=\nabla^{2} \mathrm{u}_{0}^{1}, \\
\nabla^{2} \mathrm{v}=\nabla^{2} \mathrm{v}_{0}^{1}, \\
\nabla^{2} \mathrm{w}=\nabla^{2} \mathrm{w}_{0}^{1}
\end{array}\right\}
$$

and, similarly, the divergence (4-16) of the velocity vector will reduce to

$$
\Delta_{0}^{\prime}=\frac{\partial u_{0}^{\prime}}{\partial x}+\frac{\partial v_{0}^{\prime}}{\partial y}+\frac{\partial w_{0}^{\prime}}{\partial z} .
$$

In consequence, the corresponding expressions on the right-hand sides of equations (4-10) - (4-12) are obtained if the velocity components $\mathrm{u}, \mathrm{v}, \mathrm{w}$ present there are replaced by $\mathrm{u}_{0}^{\prime}, \mathrm{v}_{0}^{\prime}, \mathrm{w}_{0}^{\prime} ;$ and $\Delta$ by $\Delta_{0}^{\prime}$.

Therefore,

$$
\begin{aligned}
\rho\left\{y \mathcal{H}-{ }_{z} \mathcal{G}\right\} & =\mu\left\{y \nabla^{2} w_{0}^{\prime}-z \nabla^{2} v_{0}^{\prime}+\frac{1}{3} D_{1} \Delta_{0}^{\prime}\right\} \\
& +\frac{\partial \mu}{\partial x}\left\{D_{1} u_{0}^{\prime}+\frac{\partial}{\partial x}\left(y w_{0}^{\prime}-z v_{0}^{\prime}\right)\right\}+
\end{aligned}
$$




$$
\begin{aligned}
& +\frac{2}{3} \frac{\partial \mu}{\partial y}\left\{2 D_{1} v_{0}^{\prime}+D_{4} w_{0}^{\prime}\right\} \\
& +\frac{2}{3} \frac{\partial \mu}{\partial z}\left\{2 D_{1} w_{0}^{\prime}-D_{4} v_{0}^{\prime}\right\} \\
& -\frac{2}{3} \frac{\partial u_{0}^{\prime}}{\partial x} D_{1} \mu+\frac{1}{3} \xi D_{4} \mu, \\
F-x \mathcal{H}\} & =\mu\left\{z \nabla^{2} u_{0}^{\prime}-x \nabla^{2} w_{0}^{\prime}+\frac{1}{3} D_{2} \Delta_{0}^{\prime}\right\} \\
& +\frac{2}{3} \frac{\partial \mu}{\partial x}\left\{2 D_{2} u_{0}^{\prime}-D_{5} w_{0}^{\prime}\right\} \\
& +\frac{\partial \mu}{\partial y}\left\{D_{2} v_{0}^{\prime}+\frac{\partial}{\partial y}\left(z u_{0}^{\prime}-x w_{0}^{\prime}\right)\right\} \\
& +\frac{2}{3} \frac{\partial \mu}{\partial z}\left\{2 D_{2} w_{0}^{\prime}+D_{5} u_{0}^{\prime}\right\} \\
& -\frac{2}{3} \frac{\partial v_{0}^{\prime}}{\partial y} D_{2} \mu+\frac{1}{3} n D_{5} \mu
\end{aligned}
$$

and

$$
\begin{aligned}
\rho\{\mathrm{x} \mathcal{G}-\mathrm{y} \mathcal{F}\} & =\mu\left\{\mathrm{x} \nabla^{2} \mathrm{v}_{0}^{\prime}-\mathrm{y} \nabla^{2} \mathrm{u}_{0}^{\prime}+\frac{1}{3} \mathrm{D}_{3} \Delta_{0}^{\prime}\right. \\
& +\frac{2}{3} \frac{\partial \mu}{\partial \mathrm{x}}\left\{2 \mathrm{D}_{3} \mathrm{u}_{0}^{\prime}+\mathrm{D}_{6} \mathrm{v}_{0}^{\prime}\right\} \\
& +\frac{2}{3} \frac{\partial \mu}{\partial \mathrm{y}}\left\{2 \mathrm{D}_{3} \mathrm{v}_{0}^{\prime}-\mathrm{D}_{6} \mathrm{u}_{0}^{\prime}\right\} \\
& +\frac{\partial \mu}{\partial z}\left\{\mathrm{D}_{3} \mathrm{w}_{0}^{\prime}+\frac{\partial}{\partial z}\left(x v_{0}^{\prime}-\mathrm{yu}_{0}^{\prime}\right)\right\} \\
& -\frac{2}{3} \frac{\partial \mathrm{w}_{0}^{\prime}}{\partial \mathrm{z}} \mathrm{D}_{3} \mu+\frac{1}{3} \zeta \mathrm{D}_{6} \mu,
\end{aligned}
$$

where the symbols $D_{j}(j=1, \ldots 6)$ stand for the following operators

$$
\begin{aligned}
& \mathrm{D}_{1} \equiv \mathrm{y} \frac{\partial}{\partial \mathrm{z}}-\mathbf{z} \frac{\partial}{\partial \mathrm{y}}, \\
& \mathrm{D}_{2} \equiv \mathbf{z} \frac{\partial}{\partial \mathrm{x}}-\mathbf{x} \frac{\partial}{\partial z},
\end{aligned}
$$




$$
\begin{aligned}
& D_{3} \equiv x \frac{\partial}{\partial y}-y \frac{\partial}{\partial x}, \\
& D_{4} \equiv z \frac{\partial}{\partial z}+y \frac{\partial}{\partial y}, \\
& D_{5} \equiv x \frac{\partial}{\partial x}+z \frac{\partial}{\partial z}, \\
& D_{6} \equiv x \frac{\partial}{\partial x}+y \frac{\partial}{\partial y} ;
\end{aligned}
$$

and where

$$
\begin{aligned}
& \xi=\frac{\partial w_{0}^{\prime}}{\partial y}-\frac{\partial v_{0}^{\prime}}{\partial z}, \\
& \eta=\frac{\partial u_{0}^{\prime}}{\partial z}-\frac{\partial w_{0}^{\prime}}{\partial x}, \\
& \zeta=\frac{\partial v_{0}^{\prime}}{\partial x}-\frac{\partial u_{0}^{\prime}}{\partial y},
\end{aligned}
$$

denote the components of vorticity of the deformation vector.

As the next step of our analysis, let us integrate both sides of the equations $(4-4)-(4-6)$ over the entire mass of our configuration with respect to the mass element

$$
d m=\rho d V=\rho d x d y d z
$$

If, as usual,

$$
\begin{aligned}
& A=\int\left(y^{2}+z^{2}\right) d m, \\
& B=\int\left(x^{2}+z^{2}\right) d m, \\
& C=\int\left(x^{2}+y^{2}\right) d m
\end{aligned}
$$

denote the moments of inertia of our configuration with respect to the 
axes $x, y, z$; and

$$
\begin{aligned}
& D=\int y z d m, \\
& E=\int x z d m, \\
& F=\int x y d m
\end{aligned}
$$

stand for the respective products of inertia, the mass integrals of the equations (4-4) - (4-6) combined with (4-1) - (4-3) will assume the forms

$$
\begin{aligned}
& A \dot{\omega}_{x}+(C-B) \omega_{y} \omega_{z}-D\left(\omega_{y}^{2}-\omega_{z}^{2}\right)-E\left(\dot{\omega}_{z}+\omega_{x} \omega_{y}\right) \\
& -F\left(\dot{\omega}_{y}-\omega_{x} \omega_{z}\right) \\
& +2 \omega_{x} \int\left(y v_{0}^{\prime}+z w_{0}^{\prime}\right) d m-2 \omega_{y} \int y u_{0}^{\prime} d m-2 \omega_{z} \int z u_{0}^{\prime} d m \\
& +\int \mathrm{D}_{1} \mathrm{PdV}-\int \mathrm{D}_{1} \Omega \mathrm{dm}=\int\left\{z(\dot{\mathrm{V}}){ }_{0}^{\prime}-\mathrm{y}(\dot{\mathrm{w}}){ }_{0}^{\prime}\right\} \mathrm{dm}+\int \rho\left\{\mathrm{y} \mathcal{H}-{ }_{\mathrm{z}} \mathcal{G}\right\} \mathrm{dV}, \\
& B \dot{\omega}_{y}+(A-C) \omega_{x} \omega_{z}-D\left(\dot{\omega}_{z}-\omega_{x} \omega_{y}\right)-E\left(\omega_{z}^{2}-\omega_{x}^{2}\right) \\
& -F\left(\dot{\omega}_{x}+\omega_{y} \omega_{z}\right) \\
& +2 \omega_{y} \int\left(x u_{0}^{\prime}+z w_{0}^{\prime}\right) d m-2 w_{z} \int z v_{0}^{\prime} d m-2 \omega_{x} \int x v^{\prime} d m \\
& +\int D_{2} P d V-\int D_{2} \Omega d m=\int\left\{x(\dot{w}) j_{0}^{\prime}-z(\dot{u}){ }_{0}^{1}\right\} d m+\int 0\{z \mathcal{G}-x \mathscr{H}\} d V,
\end{aligned}
$$

and

$$
\begin{gathered}
C \dot{\omega}_{z}+(B-A) \omega_{x} \omega_{y}-D\left(\dot{\omega}_{y}+\omega_{x} \omega_{z}\right)-E\left(\dot{\omega}_{x}-\omega_{y} \omega_{z}\right) \\
-F\left(\omega_{x}^{2}-\omega_{y}^{2}\right) \\
+2 \omega_{z} \int\left(y v_{0}^{\prime}+x u_{0}^{\prime}\right) d m-2 \omega_{x} \int_{x w_{0}^{\prime} d m-2 \omega_{y} \int y w_{0}^{\prime} d m} \\
+\int D_{3} P d v-\int D_{3} \Omega d m=\int\left\{y(\dot{u}){ }_{0}^{\prime}-x(\dot{v}){ }_{0}^{\prime}\right\} d m+\int \rho\{x \mathcal{G}-y \mathcal{G}\} d v .
\end{gathered}
$$

The preceding three equations represent the exact form of the generalized Eulerian equations governing the precession and nutation of 
self-gravitating configurations which consist of a viscous fluid. They constitute a system of three ordinary differential equations for $\omega_{x, y, z}$ considered as functions of the time $t$ alone. If the body in question were rigid (non-deformable)--or, if deformable, it were subject to no time-dependent deformation--all three velocity components $u^{\prime}, v^{\prime}, w^{\prime}$ relative to the rotating frame of reference (and thus, by (3-42), $\mathrm{u}_{0}^{\prime}, \mathrm{v}_{0}^{\prime}, \mathrm{w}_{0}^{\prime}$ ) would be identically zero. In such a case, equations (4-38) - (4-40) would reduce to their more familiar form

$$
\begin{aligned}
A \dot{\omega}_{x} & +(C-B) \omega_{y} \omega_{z}-D\left(\omega_{y}^{2}-\omega_{z}^{2}\right)-E\left(\dot{\omega}_{z}+\omega_{x} \omega_{y}\right) \\
& -F\left(\dot{\omega}_{y}-\omega_{x} \omega_{z}\right)+\int D_{1} P d V-\int D_{1} \Omega_{0} d m=\int D_{1} \Omega_{1} d m, \\
B \dot{\omega}_{y} & +(A-C) \omega_{x} \omega_{z}-D\left(\dot{\omega}_{z}-\omega_{x} \omega_{y}\right)-E\left(\omega_{z}^{2}-\omega_{x}^{2}\right) \\
& -F\left(\dot{\omega}_{x}+\omega_{y} \omega_{z}\right)+\int D_{2} P d V-\int D_{2} \Omega_{0} d m=\int D_{2} \Omega_{1} d m,
\end{aligned}
$$

and

$$
\begin{aligned}
C \dot{\omega}_{z} & +(B-A) \omega_{x} \omega_{y}-D\left(\dot{\omega}_{y}+\omega_{x} \omega_{z}\right)-E\left(\dot{\omega}_{x}-\omega_{y} \omega_{z}\right) \\
& -F\left(\omega_{x}^{2}-\omega_{y}^{2}\right)+\int D_{3} P d V-\int D_{3} \Omega_{0} d m=\int D_{3} \Omega_{1} d m
\end{aligned}
$$

where we have decomposed the total gravitational potential

$$
\Omega=\Omega_{0}+\Omega_{1}
$$

into its part arising from the mass of the respective body $\left(\Omega_{0}\right)$ and that arising from external disturbing forces $\left(\Omega_{1}\right)$ if any.

In the case of a rigid body, the existence of hydrostatic equilibrium requires that

$$
\int D_{i} P d V=\int D_{i} \Omega_{0} d m
$$


exactly for $i=1,2,3$. If, moreover, we choose our system of inertial axes $x y z$ to coincide with the principal axes of inertia of our configuration, it can be shown that all three moments of inertia (4-35) - (4-37) can be made to vanish; and for

$$
D=E=F=0
$$

our equations $(4-41)-(4-43)$ will reduce further to

$$
\begin{aligned}
& A \dot{\omega}_{x}+(C-B) \omega_{y} \omega_{z}=D_{1} \Omega_{1} d m \\
& B \dot{\omega}_{y}+(A-C) \omega_{x} \omega_{z}=D_{2} \Omega_{1} d m \\
& C \dot{\omega}_{z}+(B-A) \omega_{x} \omega_{y}=D_{3} \Omega_{1} d m
\end{aligned}
$$

which is the familiar form of the Eulerian equations for the precession of rigid bodies.

If, however, the body in question were fluid and subject to distortion by external forces--though not necessarily (like equilibrium tides) fluctuating in time--equations (4-47) would cease to be exact to the extent to which equations (4-45) need no longer hold true. The reader may note that as 1 ong as the functions $P(r)$ and $\Omega_{0}(r)$ are purely radial (as they would be in the absence of any distortion) operation with $\mathrm{D}_{\mathbf{i}}(\mathbf{i}=1,2,3)$ will annihilate them completely; so that equations (4-45) continue to be fulfilled identically. The same argument discloses, however, that for fluid bodies, equations (4-45) may become inequalities to the extent brought about by distortion; and--to this extent--the Eulerian differential equations for the precession and nutation of rigid and fluid bodies may be different even if the form of the fluid does not vary with 
the time.

If, however, this latter condition is not fulfilled--such as, for instance, in the case when the period of axial rotation of the fluid body differs from that of the revolution of an external attracting mass producing dynamical tides on the rotating fluid--the velocity components $u_{0}^{\prime}, v_{0}^{\prime}, w_{0}^{\prime}$ will emerge to give rise to supplementary terms in the equations (4-38) - (4-40) which can be classified in two groups. Those on the left-hand sides of the respective equations are factored by the angular velocity components $\omega_{x}, \omega_{y}, \omega_{z}$ which play the role of dependent variables of our problem. However, their coefficients are not constants (like A, B, C; D, E, F), but functions of the time. The second group of new terms arising on the right-hand sides of the same equations are independent of $\omega_{x, y, z}$ and render our system non-homogeneous. The first mass-integral on the right-hand sides of equations (4-38) - (4-40) arises from the accelerations $(\dot{u})_{0}^{j},(\dot{v})_{0}^{j},(\dot{w})_{0}^{j}$ experienced by the body subject to deformation--irrespective of whether the flow due to this motion is inviscid or viscous; while the second group of volume integrals (the integrands of which are given by equations (4-19) - (4-21)) represent the effects of viscosity proper; and if the latter is large, these may be predominant. 


\section{REFERENCES}

POINCARÉ, H. (1910) "Sur la précession des corps déformables," BuzZ. Astr., 27, 321-356.

OPPENHEIMER, S. (1885) "Über die Rotation und Präzession eines flüssigen Sphäroids," Astr. Nachr., 113, 209.

LIOUVILLE, J. (1858) "Développement sur un chapitre de la Mécanique de Poisson," Jourm. de Math. (2) $\underline{3}$,

GYLDÉN, H. (1871) "Recherches sur la rotation de la Terre," Publ. Univ. Obs. Uppsaza.

DARWIN, G. H. (1879) "On the Precession of a Viscous Spheroid and on the Remote History of the Earth," Phil. Trons. Roy. Soc., 170, 447-530. 